\title{
REVIEW OF BEAM DIAGNOSTICS FOR RADIOACTIVE BEAMS
}

\author{
Alberto Rovelli" \\ INFN Laboratori Nazionali del Sud, Catania, Italy
}

\begin{abstract}
Nuclear research with radioactive beams is the most recent challenge for both fundamental nuclear physics [1] and particles accelerators technologies research. Beam diagnostics is a typical component of any accelerators based facility, but for radioactive beams the technology to be used presents many analogies with that one used for standard nuclear detectors. The main reason of this peculiarity is the low, sometimes very low, intensity of the beams to be characterized but also the requirement for new kind of measurements like the unambiguous isotope identification. Another important request is the instrumentation for mass spectrometry setups where the fragments produced on the target have to be transported along a dedicated beam line as part of the detector itself. A big effort is in progress all over the word to develop new devices and techniques oriented to improve the performances of the standard beam instrumentation increasing the dynamic range in terms of energy and intensity sensitivity. Also the investigation on the use of new materials may represent a valid alternative for this improvement. In this review it will be presented the general guidelines, the problem evaluation, the requested features and the most significant ideas.
\end{abstract}

\section{INTRODUCTION}

The interest in low intensity beam diagnostics is due to the fact that a number of applications are based on the use of low intensity beams. A very important incentive for this activity came few years ago with the development of a new type of facility for the production of radioactive ion beams (RIB). The produced effort is mainly oriented to improve the performances of the conventional beam diagnostics in order to work properly also when the beam intensity is lower than $10^{8} \mathrm{pps}$. The new challenge is very hard because the required sensitivity is in the range $10^{5} \div 10^{11} \mathrm{pps}$, or even lower. The necessity to measure the beam characteristics all over such a wide intensity range and the variety of ions and energies involved forces the development of a complete set of beam instrumentation able to satisfy all the experimental requirements.

\section{REQUIREMENTS}

Several RIB facilities were proposed and realized, each one with its own characteristics [2]. For this reason it is possible to identify in the beam intensity the common problem, but the solution has to be found considering the specific procedures that are followed for the beam production, identification, acceleration and transport.

To fix the ideas, it is appropriate the effort to define an expected intensity and energy range. Starting from a general overview all around the projects it is possible to individuate the following ranges:

- beam intensity $10^{5} \div 10^{11}$ pps

- beam energy $10^{4} \div 10^{7} \mathrm{eV}$

The first problem is to individuate the right solution to develop a device able to work properly all over such wide ranges. The second one is the necessity to use the same device also when a high intensity stable beam has to be transported along the same line. Once fixed the operative ranges, it is necessary to fix also few general requirements related to the specific use of these devices. The typical parameters asked to characterize an ion beam are the current distribution, the position, the total current, the emittance and, sometime, also the time structure. A new request, typical for a RIB facility, is the unambiguous isotope identification. To perform all these measurements in a very efficient way this set of devices has to guarantee:

- the highest sensitivity to the lower current;

- the highest sensitivity to the lower energy;

- the highest reliability;

- the highest strength;

- the highest simplicity to use and to maintain it.

Furthermore, all these requirements have to fit the typical ones for standard beam diagnostics. It is evident that the ideal solution is not easy to achieve.

\section{SOLUTIONS}

There are two main guidelines that are followed approaching the low intensity beam diagnostics problem. The first one, based on the performances improvement of standard techniques, consists of a deep investigation and analysis of the typical limitations of the standard devices. The second one is based on the evaluation of using particle detection techniques very well proven for sensitivity and precision in nuclear physics research. The main problem is the difficulty to develop such a device able to cover the full range of operations. For this reason a realistic solution can be represented by two different sets of devices with an overlapping operative range.

\footnotetext{
"Email: rovelli@lns.infn.it
} 


\subsection{Standard techniques}

The number of devices nowadays available for beam diagnostic is very big and they satisfy any kind of requirements. They are based on the secondary electrons emission (wire, grids, etc.), light emission (screen, fiber, etc.), gas ionization (residual, chamber, etc.), charge induction (pick-up, transformer, etc.) and many others. The typical advantage is the simplicity in their structure and use. The main limitation is the low signal-to-noise ratio. With the exception of the ionization chambers, extensively used also as nuclear physics detectors, all the others techniques have to be subjected to a deep revision in order to increase the general performances toward the lower limits.

Two main items drive this revision. The research for new materials: higher conversion and collection efficiency, lower noise and higher radiation hardness. The electronics improvement: lower electronic noise (cables, connectors, contacts, components), lower electromagnetic noise (shielding, grounding), higher signal first amplification, higher radiation hardness. The goal is to lead the minimum sensitivity down to $10^{7} \div 10^{5} \mathrm{pps}$. In this way it will be possible to match the typical highest limit of the nuclear detectors.

\subsection{Nuclear techniques}

Typical instrumentation and techniques used in nuclear physics research can be also used for beam diagnostics if they are redesigned looking the peculiarity of this application. Typical solutions are based on:

- semiconductors;

- gas chambers;

- scintillators.

The main advantages of these devices are the sensitivity and the absolute measurements that can be performed after a suited calibration. The main limitation is the setup complexity from the point of view of its structure and use. Beam diagnostic measurements require a fast read-out of the information, on-line if possible, and at the same time well understandable by the operators. In this sense the main effort has to be devoted to:

- reduce the setup complexity;

- reduce the measuring time;

- increase the general hardness;

- increase the automated procedures.

\subsection{The ideal solution}

All these considerations allow the identification of the ideal solution. Starting from the general issue to improve the whole experimental setup it is possible to give a list of recommendations for such a device that has to be able to:

- measure different beam characteristics;

- cover a wide intensity and energy range;

- allow self calibration;

- minimize the interference with the beam;
- minimize maintenance operations and price;

- maximize reliability and versatility;

- perform charge/current collection;

- perform continuous/pulsed acquisition.

The achieved result will be as good as bigger will be the number of features satisfied. Two more general considerations have to be taken in account. Very important is the integration in the control system of the facility; this means that the operator in the console must operate the beam management with no regard to the beam intensity. Furthermore, the choice of the right device has to be done considering that the setup structure has to be strong enough to resist to quick intensity changes. If necessary, a suited interlock system (beam stop) has to be provided in order to protect the device in case of mistakes or faults.

\section{SEMICONDUCTORS}

The versatility of semiconductors, one of the most popular materials used to develop nuclear detectors, allows the realization of different configurations very useful also for beam diagnostics. In the end of this chapter it will be reported also some applications based on a particular material, the diamond. It is an insulator but it can be considered the most important alternative to the use of semiconductors.

\subsection{Silicon based detectors}

The reason of the wide use of the silicon as particles detector can be deduced from its main characteristics:

- the mean energy to produce a pair is $3.62 \mathrm{eV}$;

- well suited for different configurations;

- good timing performances;

- medium price.

Unfortunately its radiation hardness is very low. This aspect limits its use for beam diagnostics; in particular, it can be used only in single particle counting mode and, in any case, great care has to be devoted to protect it.

Silicon detectors can find useful applications for very low intensity beam diagnostics. Silicon micro-strips, for example, can be used as beam profile and position monitor. The sensitivity and the spatial resolution (higher than $100 \mu \mathrm{m}$ over a $10 \times 10 \mathrm{~cm}^{2}$ area) are very high, but the electronics and the price are very expansive. Much more suited is the application for particle identification [3]. A thin Au target is used and a silicon telescope is positioned at a suited angle in order to match a suited scattering counting rate. The $\Delta \mathrm{E}-\mathrm{E}$ information allows the ion identification; the operative energy range depends on the silicon and dead layer thickness.

\subsection{Germanium based detectors}

This very sophisticated kind of detector is mainly used for high-resolution gamma ray spectroscopy. Its main characteristics are. 
- the mean energy for a pair production is $2.96 \mathrm{eV}$;

- operating at $77^{\circ} \mathrm{K}$;

- very low radiation hardness;

- very complex experimental setup;

- very high price.

It is obvious, from this brief description, that such a detector has several limits for beam diagnostics application but, for a specific use, can be very useful. For example, it is a powerful tool for very rare radioisotopes identification [4]. The main advantage of this setup is that implanting the radio-isotope at very low energy it is possible their identification just after the production, allowing an efficient tune of the transport line avoiding any beam contamination.

\subsection{Diamond based detectors}

The operating principle of this isolating material is the same of the semiconductors one. Its main features are:

- the mean energy to produce a pair is $\sim 13 \mathrm{eV}$;

- the collection length is $50 \div 100 \mu \mathrm{m}$;

- very good radiation and power hardness;

- very good timing performances;

- versatility for different configurations;

- high price.

Nevertheless the higher energy to produce a pair, an important advantage with respect the semiconductors is the high-energy gap that strongly reduces the noise. The strength of this material allows its use with high intensity as well as low intensity beams. The very short collection length, depending on the nature and density traps, determines very high performances in terms of spatial and time resolution. Can be used in pulses counting mode, for very low beam intensities, as well in current mode looking the continuous component of the signal produced by high intensity beams. An interesting application is the use of diamond film with $100 \mu \mathrm{m}$ pitch micro-strips [5]; this setup allows the beam profile and position measurement.

The increasing interest on such a material is due to the advanced techniques nowadays available for the production of synthetic diamonds at realistic prices. The CVD (Chemical Vapor Deposition) technique allows the production of very thin diamond films of some centimeter size; the possibility to realize wide homogeneous layers with controlled impurity characteristics, justifies the big effort that is devoted to test new devices for beam diagnostics.

\section{GAS BASED DETECTORS}

Many kinds of detectors are based on the ionization produced by a charged particle crossing a gas volume. The gas can be used to fill a chamber with thin entrance and exit windows, or can be the residual gas itself contained along the beam pipes used to transport the beam.

\subsection{Gas chamber based detectors}

The most famous gas detector is the gas chamber; widely used as particle detector, it find many applications also for beam diagnostics. Its versatility, in terms of dimensions and shapes, allows the development of a variety of setups well suited for beam diagnostics applications. The signal is produced by the energy loss into the gas and its amplitude depends on the gas pressure and on the collecting electric field. The mean energy to produce a pair is about $30 \mathrm{eV}$, depending on the gas. The gas chambers have several very important features:

- very good radiation hardness;

- energy loss and charge multiplication effect;

- very good sensitivity;

- versatility for different configurations;

- medium price.

The most interesting configurations for beam diagnostics purposes are the wire chambers and the microstrips chambers [6]. Both chambers were developed to improve the sensitivity of the previous ionization chambers. The electron avalanche due to the high intensity electric field close to the anode (the wire or the micro-strip) further amplifies the signal produced by the primary ionization. The main advantages of these setups are the sensitivity and the spatial resolution. Particular interest is devoted to the micro-strips chambers because the lithographic procedure to realize the strips on a suited substrate (typically glass) allows to obtain $100 \div 200 \mu \mathrm{m}$ (the pitch) of spatial resolution. Also the chamber size can be reduced as well as the setup complexity.

Two very interesting devices were developed to measure the beam profile [7] and for particles identification [8]. Both are based on the use of a $5 \times 5 \mathrm{~cm}^{2}$ glass plate with $200 \mu \mathrm{m}$ pitch of Au strips positioned parallel with respect the beam direction and the collection field perpendicular with respect the beam direction. To measure the horizontal or the vertical beam profile the strips are parallel with respect the beam direction; for particles identification are positioned perpendicular with respect the beam direction. The whole setup structure, very simple and light, can be easily inserted or removed, through a suited actuator, to intercept the beam.

\subsection{Residual gas based detectors}

The ionization produced by the beam interaction with the residual gas contained along the beam pipes can be used to measure several beam properties without any interference with the beam itself. Generally and especially with low intensity beams, the ionization events are very rare then it is necessary some signal amplification.

The typical setup foresees a charge collecting field perpendicular with respect the beam direction and an electron amplifier, generally a micro-channel-plate (MCP), to collect the charges. 
The main advantage of this setup is that no interaction with the beam is required, for this reason it can be used without interference with the beam operations and there are no problems for the radiation damage, on condition that it is protected against the direct beam interaction. The MCP choice depends on the application; several model with different characteristics and performances are available.

In the MCP with electric readout the signal comes from the collecting electrode, the anode, is directly acquired and analyzed. This system, in different configurations, is very useful for both transversal and longitudinal beam profiles. To measure the vertical or the horizontal beam profile, as well as the beam position, the ions collecting electrode is coupled with a silicon micro-strips plate that collects the electrons coming from the previous amplification stage; the spatial resolution is very good $(0.3 \div 1 \mathrm{~mm})$. A similar setup [9], but coupled with a 50 $\Omega$ anode is used to measure the longitudinal beam profile with a very good time resolution $(100 \div 200 \mathrm{ps})$.

The operating principle of the MCP with light readout is the same of the previous one; the only difference is that the electrons coming out from the last amplification stage are accelerated and sent on a scintillating screen. The light produced by the electrons hitting the scintillator can be acquired through a common CCD camera or directly through silicon strips.

A very simple setup to measure the horizontal or vertical beam profile is based on the use of a CCD camera that collects the light coming out from a quartz window [10]. The TV signal is acquired by a frame-grabber PC board. A simple program displays the acquired image together with the beam profile and position on-line information. Regarding the camera choice is better to use a camera with gain and shutter control to match the whole setup sensitivity. A most sophisticated setup, based on the use of a thin carbon foil allows the reconstruction at the same time of both the beam profiles in the transverse plane and with higher sensitivity.

\section{SECONDARY ELECTRON EMISSION}

SEM based devices probably are the most diffused ones for beam diagnostics. The ions hitting the outer layer of several materials produce an electron emission that is proportional to the released energy. Because only the electrons contained in the first microns can exit from the material, the emission is a typical surface effect that is proportional to the surface exposed to the beam.

Moving wires, grids and thin foils are commonly used to measure several beam properties. The limitation of their use for low intensity beam diagnostics is mainly due to the bad signal-to-noise ratio. To improve their performances it is possible to devote particular care to the material selection and to the electronic noise reduction; in any case it is very difficult to increase their sensitivity more than $10^{7} \mathrm{pps}$. To do that, it is necessary to develop most sophisticated apparatus based on such an amplification (MCP, channeltron, etc.) of the detected signal [11].

\section{SCINTILLATORS}

As the previous category, also the scintillating materials are very well known and used for beam diagnostics applications. The main advantage with respect the SEM based devices is that the wide choice of materials and light detectors allows to develop several apparatus well suited also for low intensity beam diagnostics.

The first important question concerns the material choice. It is not so easy to have a global view on the scintillating materials because of their very big number, continuously in progress with the fast improvement of the technology to produce them. A significant contribute comes out also from other sectors of the scientific research where scintillating materials are employed for completely different applications. Some criteria for their selection are:

- the mean energy to produce a photon;

- the decaying time constant;

- the photon wave length;

- the refraction index of the material;

- the efficiency of photon collection;

- the radiation hardness.

Many other properties [12] have to be considered: for examples, the mechanical features as well as the hygroscope one. Furthermore, also some amorphous materials like glasses, usually doped with rare earths elements ( $\mathrm{Tb}, \mathrm{Gd}, \mathrm{Ce}$, etc.), represent an alternative choice for radiation hardness and light emission efficiency. Also for the light detector there is a wide choice of devices. Rather than a long list of the available devices (photomultipliers tubes, photodiodes, avalanche photodiodes, hybrid photodiodes, etc.) it is better to do a brief overview of the most significant applications. Profile, total current and time measurements can be easily done using scintillating optical fibers and screens.

\subsection{Beam profile and position monitor}

The simplest setup to perform beam profile and position measurement is based on the use of a scintillating screen that intercepts the beam; the emitted light is collected through a quartz window by a CCD camera and analyzed by a frame-grabber PC board. The main limitation is the bad light collection efficiency that limits the application for low intensity beams. To improve the performances it is possible the use of more efficient scintillating screens (Cr doped alumina, rare earths plastic sheets, etc.) or collecting with the same camera the light emitted at different solid angles.

A most sensitive setup to measure the beam profile was developed for very low intensities $\left(10^{4} \div 10^{6} \mathrm{pps}\right)$ and energies (higher than $10^{4} \mathrm{eV}$ ) [13]. It is based on the use of the CsI(Tl), a very performing material in terms of light 
yield. A small brick of this material is positioned behind a moving slit and it is coupled with a compact photo-tube by means of a PMMA prism. The photo-tube is completely shielded by the slit itself with respect to the beam. The electronics allows both continuos and impulsive mode acquisition. The use of faster scintillators (CsI, $\mathrm{BaF}_{2}$, etc.) increases the upper limit of the counting rate allowing absolute measurements also with normal intensities.

The scintillating fibers also offer an interesting choice for these applications. The advantage with respects the previous systems is that the efficiency in the light transmission is strongly improved. Plastics as well as glass fibers can be successful used. A very simple setup [14] is based on the same idea of the moving wires profile monitor. Sensitivity and spatial resolution depend on the fiber choice. Using glass fibers the radiation hardness is higher but the mechanical strength is lower. The light collection is performed through a compact photo-tube able to work also inside the beam pipe. A special I/V converter [15] was developed to get the continuous signal component as well as the impulsive one coming from the tube. This configuration allows to do beam measurements over the widest intensity range. Another important feature is that during the measurement it only partially intercepts the beam. The only limitations are the damage produced by the power released by the beam and the outer dead layer of the fiber (only the core is scintillating); this last problem limits its functionality at the lower energies.

\subsection{Beam time structure and current monitor}

For this kind of applications the best choice is the use of very fast organic scintillators. The high counting rate obtainable allows, if coupled with a suited photo-sensor, to get high time resolution and absolute current measurements over a wide intensity range.

To measure the phase and the phase width of pulsed beams a useful setup is based on the use of a fast plastic scintillator [16]. The PILOT-U sensor is coupled with a photo-tube through a long optical fiber; the whole setup is mounted on a radial probe to measure the beam time characteristics inside the cyclotron. The operating range is $10^{3} \div 10^{6}$ pps. The same setup can be also coupled with a silicon detector to perform $\Delta \mathrm{E}-\mathrm{E}$ measurements but at lower rates $\left(10^{3} \mathrm{pps}\right)$.

Total current absolute measurements can be performed using very fast scintillators after a suited calibration [17]. The short decay time of the polymeric plastic scintillators allows very high acquisition rates; they can be easily shaped in different geometry and are very cheap. The main drawback is their poor radiation hardness if used at low energies and high intensities.

\section{SUMMARY AND PROSPECTS}

It is not so easy to report a complete overview of the activities that are coming out developing low intensity beam diagnostics. The wide choice of materials, detectors and techniques involved produces an increasing quantity of experimental apparatus very different in terms of performances and operating ranges.

At the end of this very general overview it is possible to draw some conclusions regarding the state of art and the prospects of this activity. To satisfy all the requirements for the low intensity beam diagnostics the investigation on the use of particles detecting techniques has produced the most promising results. Gas chambers as well as scintillators based detectors represent the preferred solutions for their versatility, reliability and cost. For the next future, also the diamond based detectors will represent a good alternative. Few general statements come out from the gained experience and they can be considered as guidelines for the future setups improvements:

- necessity to reduce the setup complexity;

- necessity of integration with the control system;

- necessity to increase the dynamic range;

- necessity to improve the collaboration with the particle detecting, materials and electronics experts in order to develop new ideas, materials and electronics with better performances.

\section{REFERENCES}

[1] NuPECC Report, December 1997.

[2] J. D. Garret, Nucl. Phys. A 616 (1997) 3.

S. Kubono et al., Nucl. Phys. A 616 (1997) 11.

A. C. C. Villari, Nucl. Phys. A 616 (1997) 21.

D. Habs et al, Nucl. Phys. A 616 (1997) 29.

D. J. Morrissey, Nucl. Phys. A 616 (1997) 45.

I. Tanihata, Nucl. Phys. A 616 (1997) 56.

G. Ciavola et al, Nucl. Phys. A 616 (1997) 69.

[3] R. Pardo et al, presented at the RIB workshop, May 1997, Vancouver (Canada).

[4] B. Launé et al, presented at the RIB workshop, May 1997, Vancouver (Canada).

[5] H. Fenker et al., presented at the IEEE Nucl. Sc. Symp., November 1995, S. Francisco (CA, USA).

[6] A.Oed, NIM A 263 (1988) 351.

[7] P. Finocchiaro et al., submitted to NIM A.

[8] S. Aiello et al., NIM A 400 (1997) 469

[9] J. P. Vignet et al, AIP 390 (1996) 223.

[10] A. Rovelli et al, AIP 390 (1996) 398.

[11] D. Shapira et al, presented at the RIB workshop, May 1997, Vancouver (Canada).

[12] G. F. Knoll, Radiation Detection and Measurement, $2^{\text {nd }}$ edition.

[13] P. Finocchiaro et al., IEEE Trans. on Nucl. Sc., Vol. 45, No. 3, June 1998

[14] P. Finocchiaro et al., NIM A 385 (1997) 31.

[15] A. Amato et al., LNS Report 09-10-97.

[16] B. Launé et al, presented at the RIB workshop, May 1997, Vancouver (Canada).

[17] L. Rezzonico et al., presented at the RIB workshop, May 1997, Vancouver (Canada). 